\title{
A WATER CHERENKOV COUNTER SENSITIVE TO NONWAVESHIFTED ULTRAVIOLET CHERENKOV PHOTONS
}

\author{
C.R. BOWER, R.M. HEINZ and J. REYNOLDSON \\ Phisics Department, Indiana Unversity, Bloomington, IN 47405, USA
}

\section{S. MUFSON and J. PETRAKIS}

Astronomy Department, Indiana Unversity, Bloomington, IN 47405, USA

\author{
S.P. AHLEN, J.J. BEATTY and A. TOMASCH
}

Phystcs Department, Boston Unuersity, Boston, MA 0221.5, USA

\author{
S. BARWICK, G. GERBIER, D. LOWDER, H.S. PARK, P.B. PRICE and M.H. SALAMON \\ Phystcs Department, Unwersity of Californa, Berkeley, CA 94720, USA
}

\author{
J. MUSSER and G. TARLÉ \\ Physics Department, Unversity of Michigan, Ann Arbor, MI 48109, USA
}

Recelved 19 June 1986

\begin{abstract}
We describe a prototype water Cherenkov counter which has been bult and tested with relativistic cosmic ray muons. An analysis of the expected photoelectron yield is described. The predicted result of $315 \pm 31$ photoelectrons is compared with the experimental result of $272 \pm 30$ photoelectrons. We find that over $70 \%$ of the Cherenkov photons detected have wavelengths less than $400 \mathrm{~nm}$
\end{abstract}

\section{Introduction}

The prototype Cherenkov detector described here is a smaller version of a water Cherenkov counter which is being designed and built for a balloon-borne expenment, PBAR. The purpose of PBAR is to measure the flux of low energy ( 0.1 to $1.5 \mathrm{GeV}$ kinetic energy) antiprotons in the primary cosmic rays in order to confirm an earlier antiproton measurement [1] which could be interpreted as evidence of supersymmetric particle annihilations [2,3].

The integrated PBAR detector is a magnetic spectrometer consisting of three separate detectors: a subnanosecond time-of-flight (TOF) scintillator system; a densely packed, multilayer drift tube hodoscope located in the bore of a $15 \mathrm{kG}$, two ring superconducting magnet; and a $15 \mathrm{~cm}$ thick water Cherenkov counter in the shape of a truncated pyramid with an octagonal base $1 \mathrm{~m}$ across. The hodoscope will give the rigidity of a traversing particle and the sign of the particle's charge. The TOF scintillators will give the magnitude of the particle's charge and the particle's speed, and will reject upward going (albedo) particles. The Cherenkov coun- ter will give the speed for a particle moving faster than $0.67 \mathrm{c}$ and threshold information (no signal) for particles moving slower than this value. The redundancy of the TOF and Cherenkov signals will reduce the error in the velocity measurement when the velocity is greater than $0.67 c$ in addition to adding convincingness to the results for events below this Cherenkov threshold.

Water has been chosen for the Cherenkov radiator for several reasons. The index of refraction $(1.34$ at 400 $\mathrm{nm}$ ) provides a good Cherenkov threshold for particles whose velocity can be accurately determined by the TOF detector, and the Cherenkov signal for particles moving above this threshold aids in the velocity determination in the region where the TOF velocity resolution begins to degrade. In addition, water has a long light attenuation length, does not scintillate, is chemically inert, nonvolatile, and readily available. Also, it is unaffected by temperature changes under typical detector operating conditions. Lastly, there exists a vast literature quantifying the physical properties of water.

For the PBAR experiment we require a Cherenkov counter with a high degree of spatial uniformity of response; therefore, we considered placing the water 
radiator adjacent to (but separate from) a diffusion/integration region as had been done for previous Cherenkov counters $[4,5]$. However, the resulting detector thickness would have been awkward for its inclusion with other instruments in the limited space of a balloon gondola. For this reason we chose to place the radiator inside the light diffusion/integration region.

We also want to collect the ultraviolet Cherenkov light directly and thus avoid the need for using a waveshifter. Besides providing unwanted scintillation light, our studies showed a reduced efficiency in light yield when a waveshifter coating was placed on the photomultiplier (PM). In addition, the use of a waveshifter would add increased complexity to the photon yield analysis described in the following section. The decision to avoid using a waveshifter introduced the necessity of choosing counter components which efficiently propagate UV photons. We studied the properties of several materials in order to make these choices. The critical characteristics investigated were 1) diffuse reflectances of the light diffusion/integration region boundary, 2) effects of water purity on the light transmission, and 3) choice of the PM. Attention to detail in these areas has a significant effect on the number of photons detected and thus the ultimate resolution of the counter.

The prototype Cherenkov detector used in the current study consists of a hollow glass container in the shape of a rectangular parallelepiped with inside dimensions $18.4 \mathrm{~cm}$ high, $27 \mathrm{~cm}$ long, and $18 \mathrm{~cm}$ wide. The container is filled completely with deionized distilled water. An RCA Type 8854 PM (operated at $2150 \mathrm{~V}$ with $1000 \mathrm{~V}$ on the first stage) is mounted on the top of the counter with its face immersed in the water. The water surface that is not in contact with the PM face is surrounded by $0.64 \mathrm{~cm}$ thick TFE teflon. (The choice of the PM and of the TFE teflon will be discussed below in section 3.) Aluminium $0.16 \mathrm{~cm}$ thick is located between the teflon and the glass. An $11.7 \mathrm{~cm}$ diameter hole was cut in the top teflon and aluminum pieces in order to provide the Cherenkov photons access to the PM window.

\section{Theory and predicted response.}

A charged particle with a speed $\beta$ (relative to the speed of light) emits Cherenkov photons when traversing a medium with refractive index $n$, provided $\beta>\frac{1}{n}$. In our Cherenkov counter, some of these photons strike the photocathode of the PM and produce photoelectrons. The number of photoelectrons emitted by the photocathode which are collected by the first dynode is:

$$
\begin{aligned}
N= & \int_{200 \mathrm{~nm}}^{700 \mathrm{~nm}} \frac{2 \pi}{137} H\left(1-\frac{1}{n^{2}(\lambda) \beta^{2}}\right) \\
& \times P_{1}(\lambda) P_{2}(\lambda) \eta(\lambda) \frac{\mathrm{d} \lambda}{\lambda^{2}},
\end{aligned}
$$

where $H=$ particle's path length in water, $\lambda=$ Cherenkov photon wavelength in vacuum, $P_{1}(\lambda)=$ probability a photon is not absorbed by the water, $P_{2}(\lambda)=$ probability a photon is not absorbed by the detector walls, and $\eta(\lambda)=$ effective quantum efficiency of the PM. We assume that the fractional energy loss, $\mathrm{d} E / E$, of the emitter is negligible while it travels through the radiator and thus $\beta$ is not a function of the position of the emitter.

For our tests, we triggered on vertical, relativistic muons. Therefore $H$ is just the height of the box, 18.4 $\mathrm{cm}$, and $\beta$ is 1 . The spectral refractive index of water, $n(\lambda)$, is shown graphically in fig. 1a [6]. The emitted photon spectrum for $\beta=1$ is indicated in fig. 1b. This is
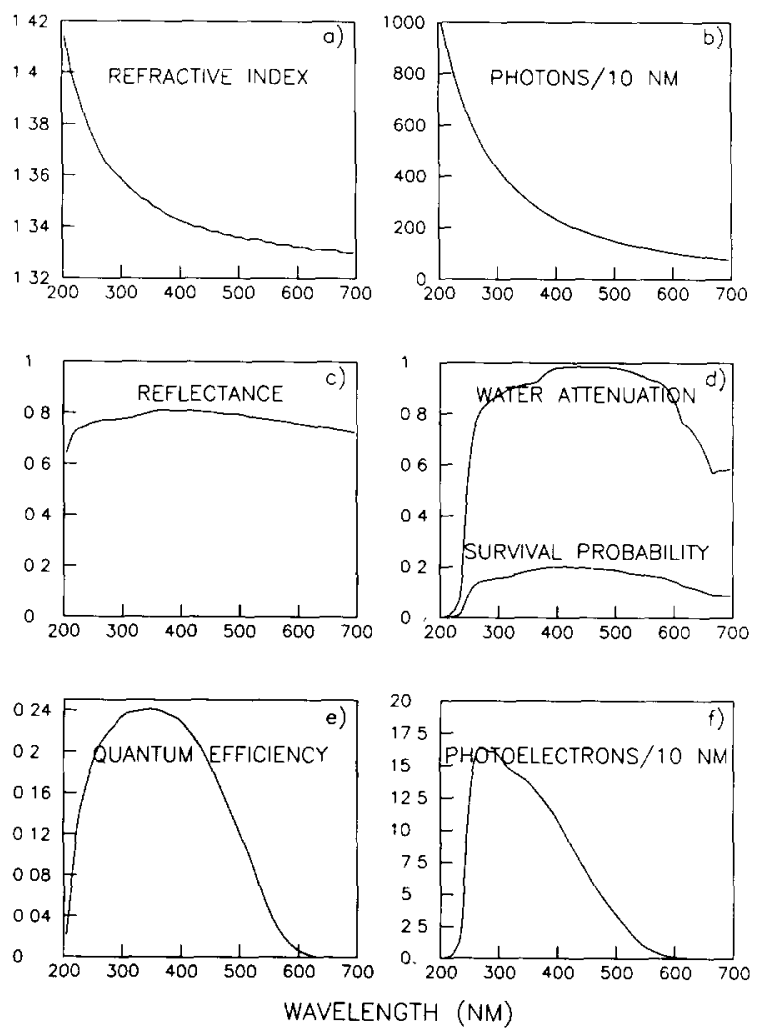

F1g. 1. a) Spectral refractive index, $n(\lambda)$, of water [6]. b) Emitted Cherenkov spectrum, displaying the $1 / \lambda^{2}$ dependence. c) Spectral reflectance, $R(\lambda)$, of $0.64 \mathrm{~cm}$ thick TFE teflon. d) (Top) Spectral light attenuation factor, $P_{1}(\lambda)$, of deionuzed distlled water and (bottom) the probability, $P_{1} P_{2}$, of a Cherenkov photon reaching the PM window. The latter quantity includes both the attenuation of the water and losses due to multiple bounces off of the teflon walls. e) Spectral effective quantum efficiency, $\eta(\lambda)$, of the particular RCA 8854 PM used in this Cherenkov counter. f) Predicted differential spectrum, $\mathrm{d} N / \mathrm{d} \lambda$, of photons which produce photoelectrons that give rise to the PM signal. This spectrum results from all of the effects shown in figs. la through 1e. The integrated area of this curve is $315 \pm 31$ photoelectrons. 
simply the integrand of eq. (1) with $P_{1}, P_{2}$, and $\eta$ all set equal to unity.

The probability, $P_{1}(\lambda)$, that a photon is not absorbed by the water is simply $\exp \left[-L / L_{0}(\lambda)\right]$ where $L$ is the average total photon path length in water and $L_{0}(\lambda)$ is the photon attenuation length of water. $L$ is found by taking the characteristic bounce length times the quantity one plus the mean number of bounces. The characteristic bounce length for our detector is $18.5 \mathrm{~cm}$. This value was calculated from an equation derived in ref. [7]. One plus the mean number of bounces is given by the expression [7]:

$1+\bar{n}_{\mathrm{b}}(\lambda)=\frac{1}{1-(1-F[1-r(\lambda)]) R(\lambda)}$,

where $F=$ the ratio of the photocathode area to the total interior surface area of the diffusion/integration region $=0.0464$ (for our detector), $R(\lambda)=$ the spectral reflectance of the diffusion/integration region boundary, and $r(\lambda)=$ the spectral reflectance of the PM window. For the TFE teflon walls of our Cherenkov detector, the diffuse reflectance, $R(\lambda)$, was measured using a Cary 14 recording spectrophotometer in reflectance mode for the wavelength range $200-700 \mathrm{~nm}$, and is shown graphically in fig. 1c. We computed a value of 0.026 for $r(400 \mathrm{~nm})$ for a water-glass interface, averaged over all angles, using Fresnel's laws of reflection. We assume that the ratio of the indices of refraction of water and glass is only weakly sensitive to wavelength between $200 \mathrm{~nm}$ and $700 \mathrm{~nm}$, and therefore use this value of $r$ for this entire wavelength range. It is worth noting that the mean photon pathlength in water is only weakly dependent on $r$ and that a value of 0.1 for $r$ increases $P_{1}$ by less than $1 \%$. Using the resulting mean total photon path length in water and the photon attenuation length of deionized distilled water [8], we calculate $P_{1}(\lambda)$. This quantity is shown graphically in the top curve of fig. $1 \mathrm{~d}$.

The probability, $P_{2}(\lambda)$, that an emitted photon is not absorbed by the reflecting walls of the diffusion/integration region is given by $P_{2}(\lambda)=\left(1+\bar{n}_{\mathrm{h}}\right) F$. (See ref. [4]). The lower curve of fig. 1d shows the photon survival probability, $P_{1} P_{2}$, which includes the combined effects of the water attenuation and the absorption of the boundary walls.

The effective quantum efficiency, $\eta$, of a PM is defined as the average number of photons detected by the PM per photon incident on the PM's window. This quantity should not be confused with either external quantum efficiency or internal quantum efficiency, which are redefined here for clarification. The photocathode external quantum efficiency is defined as the average number of electrons photoelectrically emitted from the photocathode per photon incident upon the external side of the PM window [9]. The photocathode internal quantum efficiency is defined as the average number of electrons photoelectrically emitted from the photocathode per photon incident upon the photocathode [9]. External quantum efficiency is thus the product of the internal quantum efficiency and the transmission of the PM window. $\eta$ also includes the first dynode collection efficiency, that is the average number of photoelectrons which reach the first dynode per photoelectron which leaves the photocathode. $\eta$ is the external quantum efficiency times the first dynode collection efficiency.

The photocathode blue luminous responsivity measurement (usually performed by the manufacturer for each PM immediately before shipment, with results attached to the shipping container) can be used to calculate $\eta$ for a particular PM. The conversion from photocathode blue luminous responsivity to effective quantum efficiency at the blue filter's peak wavelength is given by:

$\eta\left(\lambda_{\text {bluc }}\right)=\frac{1240}{\lambda_{\text {blue }}} S_{\text {bluc }} f_{1} f_{2}$,

where $\lambda_{\text {bluc }}=$ wavelength (expressed in $\mathrm{nm}$ ) of maximum transmission of the blue filter, $S_{\text {hlue }}=$ photocathode blue luminous responsivity (expressed in units of amperes per lumen), $f_{1}=$ factor for converting blue luminous responsivity to total luminous responsivity (that is, the response to the same light source without the blue filter present), and $f_{2}=$ factor (expressed in units of lumens per watt) for converting total luminous responsivity to total radiant responsivity. The above quantities depend on the spectral intensity of the light source, the spectral transmittance of the blue filter, and the PM window-photocathode combination. The conversion factors $f_{1}$ and $f_{2}$ are often found in the data booklet for the given PM type and can be obtained from the manufacturer.

$S_{\text {blue }}$ for the particular RCA 8854 PM used in our Cherenkov detector is $8.5 \times 10^{-6} \mathrm{~A} / \mathrm{lm}$, measured by the manufacturer under the following conditions: tungsten filament light source at a color temperature of $2870 \mathrm{~K}$, a flux of $100 \mu \mathrm{lm}$ incident upon a Corning C.S. No. 5-58 filter polished to $1 / 2$ stock thickness, and a potential of $500 \mathrm{~V}$ between the cathode and all other electrodes connected together as the anode. For the RCA type $8854 \mathrm{PM}, f_{1}=7.7$ and $f_{2}=1140 \mathrm{~lm} / \mathrm{W}$ [10] Using these values and the above equation, we derive a value for $\eta$ of $23 \%$ at $400 \mathrm{~nm}$. This value of quantum efficiency applies for photons incident normal to the PM window and coming through air. In our tests, the photons arrived from a random direction through water. Photons which arrive with a nonzero angle of incidence are more likely to be reflected than photons which arrive normal to the window. However, a water-glass interface has higher transmittance than an air-glass interface so these two effects tend to cancel. Again we used Fresnel's laws to derive the mean transmittance of 
the PM window under these different conditions. Our calculations show that there is an enhancement of the effective quantum efficiency by a factor of 1.03 for our test conditions as compared to the manufacturers test conditions mentioned above. We normalize the spectral response characteristics curve of a typical RCA 8854 [10] to $\eta(400 \mathrm{~nm})=0.24$, resulting in the curve shown in fig. le.

Using the values from figs. 1a through $1 \mathrm{e}$, eq. (1) predicts a mean signal of $315 \pm 31$ photoelectrons for a vertical, relativistic, singly charged particle passing through our detector. The uncertainty in this prediction is primarily due to the uncertainty in $R(\lambda)$. The differential distribution of photon wavelengths giving rise to these photoelectrons is shown in fig. 1f.

\section{Cherenkov counter components.}

Before constructing the Cherenkov counter, we investigated the diffuse reflectances of several substances for possible use as the boundary for the diffusion/integration region. In order to maximize $P_{2}(\lambda)$, this reflectance must be high. A water-based barium sulfate paint has been produced which has a mean reflectance of nearly $97 \%$ in the $300-600 \mathrm{~nm}$ wavelength range [4]. However, in our design the light diffusion/integration region is filled with water. Water-based barium sulfate paint flakes off of a surface immersed in water, so we were not able to use it in our detector.

We developed oil-based and lacquer-based barium sulfate paints, but test samples of these were also damaged in water. In addition, spectrophotometer analyses showed these samples to be greatly reduced in reflectance. We also tried to find a clear plastic to place over all interior surfaces to protect the barium sulfate, but even ultraviolet-transmitting (UVT) lucite absorbed far too much in the ultraviolet. Only TFE teflon, of the diffusely reflecting surfaces tested, was found to be highly reflective across wavelengths ranging from 200 to $700 \mathrm{~nm}$ and to be unaffected by contact with water. The spectral reflectance of the $0.64 \mathrm{~cm}$ thick TFE teflon used in our counter is shown in fig. 1c.

We compared results of vertical, relativistic muon tests with deionized and nondeionized distilled water and found that the latter results in $20 \%$ fewer photoelectrons per signal. This observation is consistent with a previous investigation [8] which showed a significant increase for the ultraviolet attenuation length of deionized distilled water over that of distilled water.

The choice of the PM also affected the quality of the Cherenkov detector. We compared the results of the RCA 8854 with an RCA 4522 which had a UVT lucite faceplate attached. The 8854 and 4522 are otherwise identical except for the first dynode, which is composed of high gain GaP for the 8854 and $\mathrm{BeO}$ for the 4522 . The typical muon signal for the 4522 was less than $50 \%$ of the 8854 signal. This result can be attributed to the poor transmitting properties of UVT lucite for photons with wavelengths less than 350 nanometers and emphasizes the value of the Corning 9741 UVT glass window of the 8854 . In addition, the high gain first

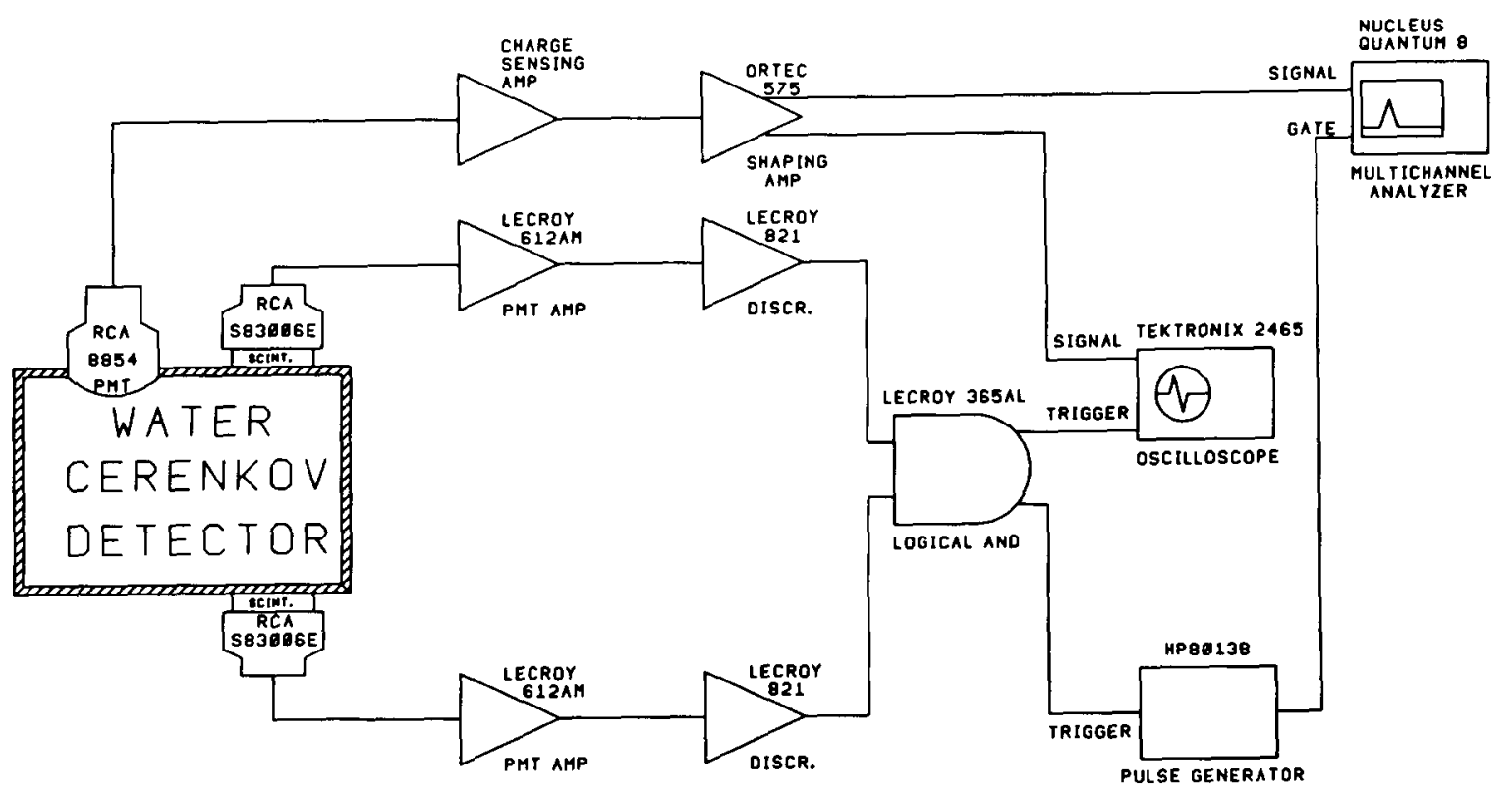

Fig. 2. Diagram of the experimental setup for the tests measuring the number of photoelectrons detected per vertical sea level cosmic ray muon. 
dynode of the 8854 (a gain of 50 for a potential of 1000 $\mathrm{V}$ [11]) reduces the resultant $\mathrm{PM}$ output fluctuations considerably over those of the low gain first dynode of the 4522 (a gain of 8 for a potentral of $570 \mathrm{~V}$ [11]).

\section{Experimental setup}

A diagram of the experiment's electronics setup is shown in fig. 2. Two RCA S83006E PM's, each with a square of plastic scintillators against its face, were used as a trigger system for the experiment. One was placed above the Cherenkov detector and the second below, in a vertical alignment. The output of each trigger PM was amplified by a LeCroy 612AM linear amplifier with a gain of 4 . Each signal was then passed through a LeCroy 821 discriminator with a threshold set at $40 \mathrm{mV}$ (1/2 muon level). The discriminated signals were fed through a LeCroy 365AL logic unit in the AND mode to determine coincidence. This coincidence signal was then used to trigger both an oscilloscope and a square pulse generator. The pulse generator's output was used to gate a multichannel pulse height analyzer (PHA).

The output signal of the Cherenkov PM was passed through a charge integrating amplifier. The output voltage pulse of this amplifier (rise time $=0.15 \mu \mathrm{s}$, exponential fall time $=4.5 \mu \mathrm{s}$ ) was input to a LeCroy 575 shaping amplifier in positive first bipolar mode set on a gain of five. This output was then sent to the oscilloscope and to the PHA.

\section{Experimental results}

Fig. 3a shows our resulting muon peak for a $65 \mathrm{~h}$ run. The width of this peak exhibits several physical effects besides photoelectron fluctuations. Because of the nonnegligible horizontal extent of the trigger scintillators, not all of the muons are vertical. These nonvertical muons have a larger path length through the water (radiator) and the resulting signal is equal to the vertical muon signal times the secant of the particle's zenith angle. Nonuniform spatial response for the light collected from particles entering the box at different locations could also cause signal fluctuations. Another broadening effect is due to the fact that not all of the Cherenkov emitters are relativistic muons. The vast majority of sea level muons are created high in the atmosphere. Since the muon lifetime is short, those which are strongly time dilated (and thus have $\beta$ near 1) survive long enough to pass through our detector. Over $90 \%$ of sea level cosmic ray muons have $\beta>0.98$ [12]. The few $(<10 \%)$ slower moving muons give off less light (as can be seen from eq. (1)), causing the muon PHA distribution below the peak to become enhanced. Other broadening effects are from the production of

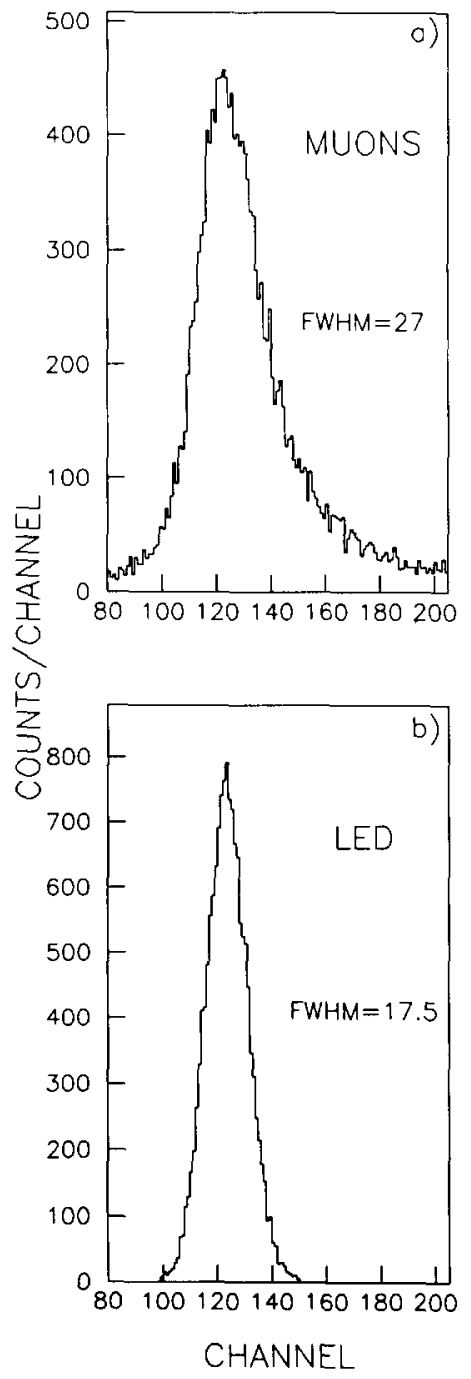

Fig. 3. a) The cosmic ray muon Cherenkov pulse height spectrum for a 65 hour run. b) The pulse height spectrum of a green LED whose peak (in channel 122) has been matched to the peak of the muon pulse height spectrum of fig. $3 a$.

relativistic knock-on electrons (delta-rays) by the Cherenkov emitter (which probably cause the "Landau type" tail apparent in fig. 3a) and the occurrence of multiple particle events (showers).

In order to filter out these broadening effects, a green light-emitting-diode (LED) was used to simulate the Cherenkov light from a vertical, relativistic muon. The LED was driven by an HP 8013B pulse generator. The full width at half maximum (fwhm) of the voltage pulse was $5.5 \mathrm{~ns}$ and its frequency was $200 \mathrm{~Hz}$. The pulse amplitude was adjusted until the peak channel of the LED signal (collected by the PM and displayed on the PHA) was equal to the peak channel of the muon pulse height spectrum. The resulting LED PHA spec- 
trum is shown in fig. $3 \mathrm{~b}$. We assume that the fluctuation of the number of photons given off by the LED is negligible compared to the PM's photoelectron fluctuations.

Two independent methods are used to determine the mean number of LED-generated photoelectrons. The first measurement technique involves comparıng the smoothed oscilloscope traces of the anode pulses from the single photoelectron (dark) noise of the RCA 8854 and from the LED signal. These signals are shown in figs. $4 a$ and $4 b$, respectively. Since the PM measures charge delivered to the anode, the ratio of the integrated areas of these two curves is the number of photoelectrons arriving at the first dynode. In order to draw this conclusion, we assume that the tube gain is identical for both the LED signal and the single photoelectron pulse; in other words, we assume that the tube output is linear.

For this first technique, the divider network potential was increased to $2450 \mathrm{~V}$ before recording either oscilloscope pulse in order to see the unamplified single photoelectron signal. This change should not influence the LED signal measurement since the external quantum efficiency is not affected by the divider voltage and the cathode to dynode- 1 potential was maintained at $1000 \mathrm{~V}$ with zener diodes, thus elıminating the possibil-
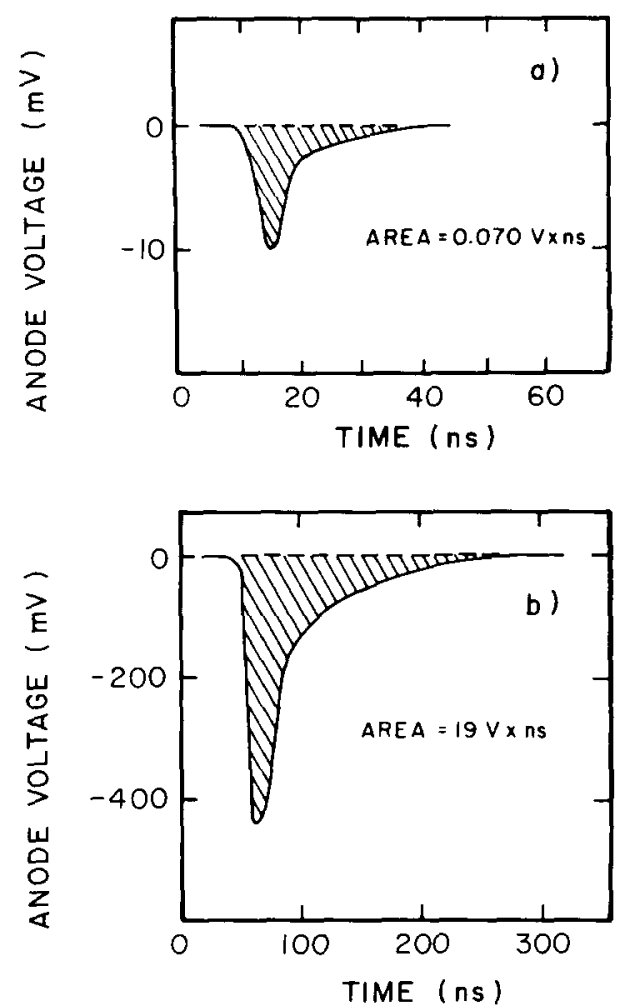

Fig. 4. a) The smoothed anode pulse of a single (dark noise) photoelectron signal. b) The smoothed anode pulse of a typical LED flash which gives rise to the muon pulse height spectrum of fig. $3 \mathrm{~b}$. ity of varying the first dynode collection efficiency. The result of this measurement was $270 \pm 30$ photoelectrons. The uncertainty reflects the difficulty in reading the oscilloscope trace due to the fluctuations of each PM signal. This technique could suffer from nonlinearity of the PM output due to space charge effects between the last two dynodes. We did observe this phenomenon at voltages above $2450 \mathrm{~V}$. Space charge effects at $2450 \mathrm{~V}$ were determıned to be negligible by comparing an LED signal from this PM and divider network combination with the signal from the same PM but with a different (tapered) divider network. The voltage on this second divider network was varied from 2330 to $2430 \mathrm{~V}$ to verify that it was not suffering from space charge effects. The output levels (measured in number of photoelectrons) for the two different divider networks were observed to be equal within experimental error.

The second technique used to determine the mean number of photoelectrons involves measuring the width of the LED pulse height spectrum and converting this to a mean number of photoelectrons. We attribute this width to statistical fluctuations in the number of photoelectrons collected by the first dynode of the PM. A rigorous derivation of $\mathrm{PM}$ signal-to-noise ratio can be found in ref. [13]. They derive:

$\sigma_{\mathrm{dc}}^{2}=\frac{G^{2}}{\delta_{1}}, \quad \bar{n}_{\mathrm{pe}}=\eta \bar{n}_{\mathrm{p}}$,

$\bar{n}_{\mathrm{PM}}=\eta \bar{n}_{\mathrm{P}} G, \quad \sigma_{\mathrm{PM}}^{2}=\eta \bar{n}_{\mathrm{p}}\left(G^{2}+\sigma_{\mathrm{dc}}^{2}\right)$,

where $\sigma_{\mathrm{dc}}^{2}=$ the variance of the output signal of the dynode chain, $G=$ the current amplification of the PM, $\delta_{1}=$ the gain of the first dynode, $\bar{n}_{\mathrm{pe}}=$ the mean number of photoelectrons reaching the first dynode, $\bar{n}_{\mathrm{p}}=$ the mean number of photons striking the PM window, $\bar{n}_{\mathrm{PM}}=$ the mean number of electrons collected at the anode, and $\sigma_{\mathrm{PM}}^{2}=$ the variance of the output signal of the PM.

The first of these equations assumes that the PM's first stage has a very large gain and exhibits Poisson statistics. (Both of these assumptions hold for the GaP first dynode of the RCA 8854 PM [13].) These equations also assume that the emission of photoelectrons from the photocathode is a Poisson process. Combining these equations, substituting fwhm $_{\mathrm{PM}} / 2.355$ for $\sigma_{\mathrm{PM}}$, and solving for $\bar{n}_{\mathrm{pe}}$ gives:

$\bar{n}_{\mathrm{pe}}=\left(2.355 \frac{\bar{n}_{\mathrm{PM}}}{\text { fwhm }_{\mathrm{PM}}}\right)^{2}\left(1+\frac{1}{\delta_{1}}\right)$.

Assuming that the amplifiers and PHA introduce no fluctuations into the signal, it follows that:

$\bar{n}_{\mathrm{pc}}=\left(2.355 \frac{\bar{s}_{\mathrm{PHA}}}{\mathrm{fwhm}_{\mathrm{PHA}}}\right)^{2}\left(1+\frac{1}{\delta_{1}}\right)$.

where $\bar{s}_{\text {PHA }}$ refers to the PHA channel corresponding to the peak of the LED signal distribution. 
Using the value of 50 for $\delta_{1}[11]$ and the values of 122 for $\bar{s}_{\mathrm{PHA}}$ and 17.5 for fwhm ${ }_{\mathrm{PHA}}$ from fig. $3 \mathrm{~b}$, we obtain a result of $275 \pm 30$ photoelectrons. This number agrees with the anode pulse measurement described above. The uncertainty is primarily due to the error in determining $\mathrm{fwhm}_{\mathrm{PHA}}$. This uncertainty is estimated by measuring the variation of $f w h m_{\mathrm{PHA}}$ from several LED PHA spectra, each with its peak in channel 122.

\section{Conclusion}

A water Cherenkov counter utilizing TFE teflon as the diffuse reflecting surface has been constructed and tested. The water radiator is located in the light diffusion/integration region. An analysis of the photon production, transmission, reflection, and absorption is performed in order to predict the photoelectron yield of an RCA 8854 PM. The prediction of $315 \pm 31$ photoelectrons is compared with the experimental result of $272 \pm$ 30 photoelectrons. This favorable agreement lends support to the analysis and thereby shows the importance of detecting the near ultraviolet $(250-400 \mathrm{~nm})$ Cherenkov photons.

\section{References}

[1] A Buffington, S M. Schindler, and C.R. Pennypacker, Astrophys J. 248 (1981) 1179.
[2] J Silk, and M. Srednickı, Phys. Rev Lett. 53 (1984) 624

[3] F W. Stecker, S Rudaz, and T. Walsh, Proc 19th Int Cosmic Ray Conf. 2 (La Jolla, 1985) 358.

[4] S.P Ahlen, B.G Cartwright, and G. Tarlé, Nucl Instr. and Meth. 143 (1977) 513.

[5] S.P Ahlen, P B Price, M.H. Salamon, and G. Tarlé, Nucl Instr. and Meth. 197 (1982) 485

[6] H S. Stewart and R.F Hopfield, Appled Optics and Optical Engineernng I, ed., R. Kingslake (Academic Press, New York, 1975) p. 151

[7] S.P. Ahlen, Ph.D Thesis (University of Calıfornia, Berkeley, 1976)

[8] R.M. Bionta, C.B. Bratton, B G. Cortez, S. Errede, G W Foster, W. Gajewski, M Goldhaber, J. Greenberg, T W. Jones, W.R Kropp, J Learned, J.M LoSecco, P.V. Ramana Murthy, H.S. Park, F. Reınes, J Schultz, E Shumard, D Sinclair, D.W. Smith. H. Sobel, J L Stone, L.R Sulak, J.C. van der Velde, and C Wuest, Proc. 17th Rencontres de Moriond 1 (Savole, France, 1982) 447.

[9] W. Budde, Physical Detectors of Optical Radiation (Academic Press, New York, 1983) p 11

[10] RCA 8854 Photomultiplier Data Booklet (RCA Solid State Division, Lancaster, PA, 1971).

[11] R.W Engstrom, RCA Photomultiplier Handbook (RCA, Lancaster, PA; 1980) pp. 22-23.

[12] A W Wolfendale, Cosmic Rays (Philosophical Library, New York, 1963) p. 142.

[13] ref. [11], pp 166-9 